
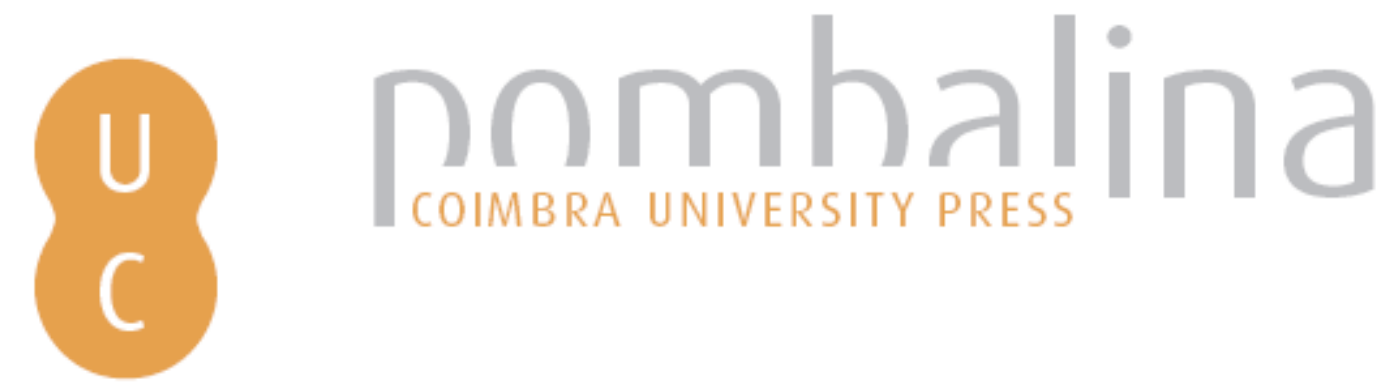

\title{
Aplicaciones de radiación gamma y UV para el injerto molecular y el diseño de matrices para aplicaciones terapéuticas.
}

Autor(es): $\quad$ Meléndez Ortiz, Héctor I.; Burillo, Guillermina; Bucio, Emilio

Publicado por: Imprensa da Universidade de Coimbra

URL

persistente: URI:http://hdl.handle.net/10316.2/36877

DOI: $\quad$ DOI:http://dx.doi.org/10.14195/978-989-26-0881-5_11

Accessed : $\quad$ 26-Apr-2023 14:07:00

A navegação consulta e descarregamento dos títulos inseridos nas Bibliotecas Digitais UC Digitalis, UC Pombalina e UC Impactum, pressupõem a aceitação plena e sem reservas dos Termos e Condições de Uso destas Bibliotecas Digitais, disponíveis em https://digitalis.uc.pt/pt-pt/termos.

Conforme exposto nos referidos Termos e Condições de Uso, o descarregamento de títulos de acesso restrito requer uma licença válida de autorização devendo o utilizador aceder ao(s) documento(s) a partir de um endereço de IP da instituição detentora da supramencionada licença.

Ao utilizador é apenas permitido o descarregamento para uso pessoal, pelo que o emprego do(s) título(s) descarregado(s) para outro fim, designadamente comercial, carece de autorização do respetivo autor ou editor da obra.

Na medida em que todas as obras da UC Digitalis se encontram protegidas pelo Código do Direito de Autor e Direitos Conexos e demais legislação aplicável, toda a cópia, parcial ou total, deste documento, nos casos em que é legalmente admitida, deverá conter ou fazer-se acompanhar por este aviso.

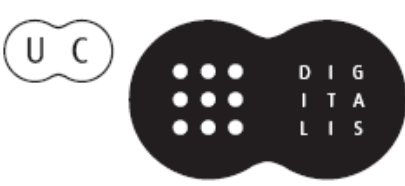


IOMATERIAIS APLICADOS AO DESENVOLVIMENTO DE SISTEMAS TERAPÊUTICOS AVANÇADOS

IOMATERIALES APLICADOS AL DISEÑO DE SISTEMAS TERAPÉUTICOS AVANZADOS

Hermínio C. de Sousa Mara E. M. Braga Alejandro Sosnik (editores) 


\title{
CAPÍTULO 11.APLICACIONES DERADIACIÓN GAMMA Y UUV PARA EL INJERTO MOLECULAR Y EL DISEÑO DE MATRICES PARA APLICACIONES T E R A P É UT I C A S
}

\begin{abstract}
Héctor I. Meléndez Ortiz, Guillermina Burillo, Emilio Bucio Centro de Investigación en Química Aplicada, Saltillo, México Departamento de Química de Radiaciones y Radioquímica, Instituto de Ciencias Nucleares, Universidad Nacional Autónoma de México, Circuito Exterior, Ciudad Universitaria,
\end{abstract} 04510 México DF, México.

\section{Resumen:}

Los polímeros han comenzado a ser prevalentes en muchos productos comerciales debido a su fácil manufactura y su relativo bajo costo (comparado con productos metálicos y cerámicos). Las propiedades de estos materiales pueden ser adaptadas mediante la copolimerización con otros polímeros para darles una gran variedad de usos. La copolimerización mediante rayos gamma resulta un interesante método para modificar matrices poliméricas de interés biomédico. Esta técnica ha sido empleada para funcionalizar las superficies de polímeros con diferentes grupos funcionales así como también para injertar nuevas cadenas de polímero sobre los existentes materiales funcionalizados con el objetivo de alterar sus propiedades. Generalmente, la polimerización mediante radiación gamma procede mediante el mecanismo por radicales libres pero sin el uso de iniciadores químicos. Los métodos de injerto normalmente incluyen el método de pre-irradiación oxidativa así como el método directo. Este capítulo 
describe las diferentes técnicas de injerto y los efectos de varios parámetros en las reacciones de polimerización de injerto iniciada con radiación gamma. Además, describe las diferentes técnicas analíticas usadas para la caracterización de copolímeros de injerto.

Palabras clave: Radiación gamma; métodos de injerto; pre-irradiación; copolímeros; caracterización de polímeros.

\begin{abstract}
:
Polymers have become prevalent in many commercial products due to their easy manufacture and relative low cost (compared with metals and ceramic products). The properties of these materials can be tailored by copolymerization with others polymers in order to give them a variety of engineering and scientific uses. Copolymerization via gamma-rays is an interesting method to modify polymeric matrices of biomedical interest. It has been used to functionalize the surfaces of polymers with different chemical groups, as well as to graft new polymer chains onto existing functional materials to alter their properties. Usually, polymerization using gamma-rays proceeds via the free radical initiation mechanism but without the use of chemical initiators. Grafting methods normally include the oxidative preirradiation, as well as the mutual or the simultaneous method. This chapter describes the different grafting techniques and the effect of various parameters in the grafting polymerization initiated with gamma radiation. Also, it describes the different analytical techniques used for the characterization of graft copolymers.
\end{abstract}

Keywords: gamma radiation; grafting methods; preirradiation; copolymers; polymer characterization. 


\subsection{Introducción}

Recientemente ha cobrado una gran relevancia la modificación de polímeros en la superficie mediante métodos químicos y radiación ionizante: electrones acelerados y rayos gamma. Esta modificación se puede llevar a cabo mediante un fotoiniciador (en el caso de radiación UV) o mediante radiación ionizante empleando un disolvente que penetre (se absorba) solo en la superficie del polímero. La modificación de superficies en materiales poliméricos es una técnica indispensable ya que se pueden obtener materiales con mejores propiedades mecánicas; así como tambien permite la funcionalización de polímeros con grupos de interés, biomoléculas e inmovilización de enzimas, etc.

Por ejemplo, la inmovilización por medio de membranas tiene numerosas aplicaciones, tales como, liberación controlada de fármacos en el cuerpo humano, cromatografía, producción de órganos artificiales, inmovilización de lípidos en membranas o sólidos, etc. En resumen, la modificación superficial de matrices poliméricas tiene una amplia gama de aplicaciones tanto industriales como biológicas.

Para obtener películas modificadas en la superficie o copolímeros de injerto, existen varios tipos de radiación: fotónica (radiación UV) y radiación ionizante (por ejemplo, la radiación gamma y electrones acelerados). La radiación gamma es utilizada para estudios fundamentales y para irradiación a dosis bajas y de alta penetración de materiales poliméricos. Por otro lado, la irradiación por electrones se obtiene generalmente por medio de aceleradores y la penetración correspondiente es de solo unos milímetros. Entre otros tipos de irradiación se pueden encontrar los rayos $\mathrm{X}$, fotones, partículas alfa, etc.

Ente los métodos de irradiación con rayos gamma, se consideran los siguientes por su disponibilidad: i) irradiación directa, en este método, se coloca la matriz polimérica que se desea injertar en una ampolleta de vidrio y se agrega una concentración conocida de solución monómero-disolvente. Se elimina él oxigeno del sistema, se sella la ampolleta al vacío y finalmente es irradiada. Por este método algunas veces predomina la homopolimerización sobre la copolimerización cuando el monómero es muy reactivo y para 
evitar este problema usualmente se agregan sales inorgánicas que actúan como inhibidores de la homopolimerización. ii) pre-irradiación, en este caso, la matriz polimérica se irradia previamente a una dosis e intensidad deseada, posteriormente se coloca la matriz en una ampolleta de vidrio, la cual contiene una solución monómero-disolvente, en la cual se elimina el oxígeno y es sellada al vacío. Finalmente la ampolleta se coloca en un baño a temperatura controlada para dar paso a la copolimerización.

\subsection{Macromoléculas}

\subsubsection{Polímeros}

Los polímeros naturales y sintéticos son moléculas esenciales para nuestra existencia y bienestar ya que son constituyentes de nuestra comida (almidón, proteínas, etc.) y en nuestra vida diaria (electrodomésticos, muebles, computadoras, automóviles etc.). Los polímeros se producen por la unión en secuencia de cientos de miles de moléculas denominadas monómeros, una unidad después de la otra, para formar largas cadenas de las formas más diversas.

\subsubsection{Copolímeros}

Cuando un polímero se forma por medio de uniones entre sí de un solo monómero, se le conoce como homopolímero, mientras que un copolímero es una macromolécula compuesta por dos o más unidades repetitivas distintas, que se pueden unir de diferentes formas por medio de enlaces químicos. Estas combinaciones de monómeros se realizan para modificar las propiedades de los polímeros y lograr nuevas aplicaciones. Al variar las proporciones de los monómeros, las propiedades de los copolímeros varían también de manera que el proceso de copolimerización permite hasta cierto punto sintetizar polímeros con propiedades y características deseadas. 


\subsubsection{Copolímeros alternados}

Cuando los dos monómeros están dispuestos según un ordenamiento alternado, el polímero es denominado copolímero alternado (Figura 11.1).

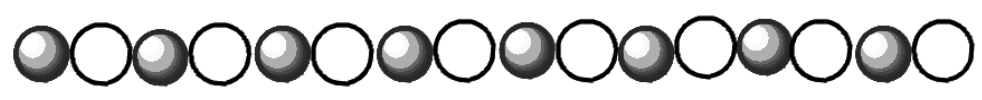

Figura 11.1. Representación de un copolímero alternado.

\subsubsection{Copolímeros al azar}

En un copolímero al azar, los dos monómeros pueden seguir cualquier orden (Figura 11.2).

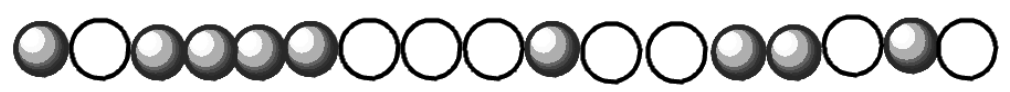

Figura 11.2. Representación de un copolímero al azar.

\subsubsection{Copolímeros de bloque}

En un copolímero de bloque, las cadenas poliméricas están formadas por largas secuencias (bloques) de una misma unidad monomérica covalentemente unidas a otra larga secuencia de otra unidad monomérica diferente. Los bloques pueden estar conectados en diversas formas, como por ejemplo dibloques o tribloques. Un copolímero de bloque puede ser imaginado como dos homopolímeros unidos por sus extremos (Figura 11.3).

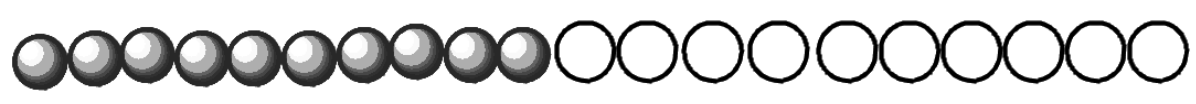

Figura 11.3. Representación de un copolímero en bloques. 


\subsection{Injertos inducidos por radiación ionizante}

Debido a que los copolímeros son el resultado de la combinación química de dos moléculas de diferente naturaleza y que las radiaciones son creadoras de sitios activos en los polímeros, esto nos conduce a numerosos procesos de química de radiaciones en macromoléculas, dando como resultado copolímeros de injerto. El injerto inducido por radiación es un método bien conocido, el cual ha sido reportado desde la década de los 60's [1-3]. Este se puede obtener en substratos tales como fibras o películas, con un monómero en fase líquida, vapor o solución. Actualmente existen cuatro métodos para obtener injertos mediante radiación: preirradiación, pre-irradiación oxidativa, irradiación directa e irradiación directa en fase vapor.

\subsubsection{Copolímeros de injerto}

Se tiene un copolímero de injerto cuando las cadenas de un polímero formado a partir de un monómero se encuentran injertadas en una matriz polimérica (Figura 11.4). Una característica de estos copolímeros es que conservan las propiedades de los polímeros que lo componen.

La síntesis de un copolímero de injerto requiere la formación de un centro reactivo sobre una matriz polimérica en la presencia de un monómero polimerizable. La mayoría de los métodos para sintetizar copolímeros de injerto involucran la polimerización vía radicales libres. La copolimerización mediante injerto puede ser llevada a cabo en un sistema homogéneo ó heterogéneo dependiendo si la matriz polimérica es soluble o insoluble en el monómero y disolvente empleados. 


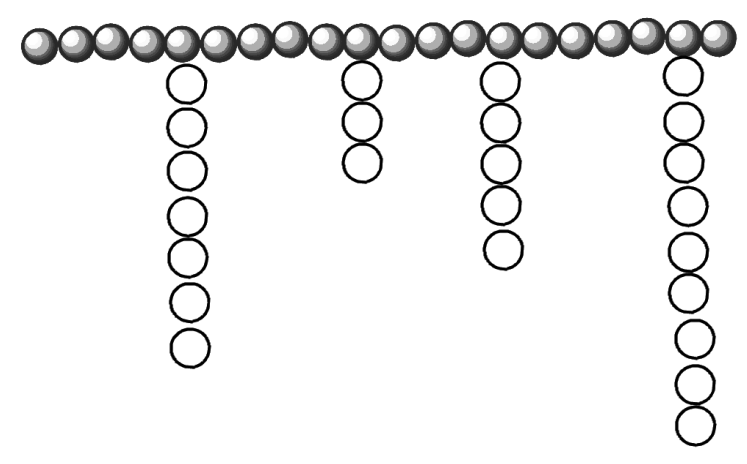

Figura 11.4. Representación de un copolímero de injerto.

El injerto puede proceder como se ilustra a continuación:

a) Un sitio activo generado sobre la matriz polimérica $\left(\mathrm{P}_{1}\right)$ inicia la etapa de propagación agregando moléculas de monómero (M) y de esta manera formar el copolímero de injerto (Figura 11.5).

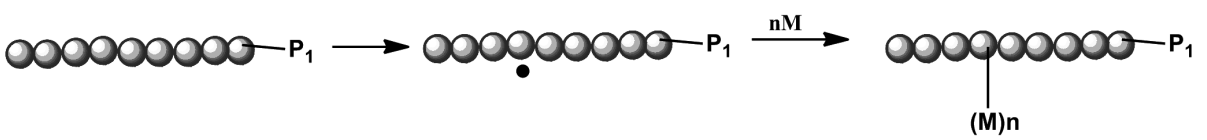

Figura 11.5. Generación de radicales libres sobre la matriz polimérica y su propagación.

b) Una cadena polimérica en la etapa de propagación $\left(\mathrm{P}_{2}\right)$ que reacciona con el radical libre formado sobre la matriz polimérica $\left(\mathrm{P}_{1}\right)$ (Figura 11.6).

c) Una etapa de terminación mediante combinación de los radicales en propagación o mediante una reacción de desproporción. 

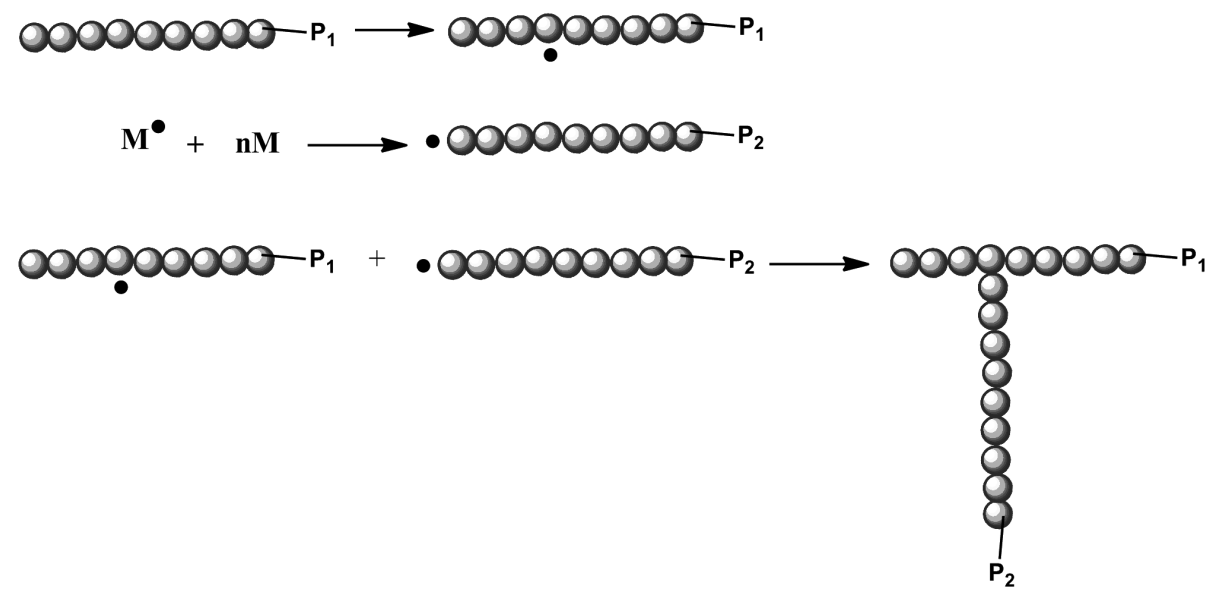

Figura 11.6. Etapa de propagación en la preparación de copolímeros de injerto.

Durante la síntesis de un copolímero de injerto se asume que: los injertos sobre la matriz polimérica son al azar [4-6]; cada segmento de la matriz polimérica tiene la misma probabilidad de ser injertada (un segmento es definido como la parte de la matriz polimérica en la cual solo un injerto puede ser llevado a cabo); la probabilidad de que ocurra un nuevo injerto sobre la matriz polimérica no es afectada por las cadenas poliméricas injertadas previamente.

La eficiencia del injerto depende de las reacciones en competencia por el radical iniciador entre el monómero, disolvente y la matriz polimérica. Además, si la terminación ocurre a través de dos cadenas poliméricas en crecimiento, la eficiencia de injerto disminuye.

El grado de esas transformaciones depende de la naturaleza del polímero, de las condiciones de reacción, así como del tratamiento que se le dé antes y después de la irradiación [7-9].

\subsubsection{Ventajas de los copolímeros de injerto sintetizados mediante radiación ionizante}

i) El número y longitud de las cadenas injertadas pueden ser controlados seleccionando cuidadosamente la dosis e intensidad de dosis de irradiación. 
ii) No se requiere iniciador químico, por lo que no es necesaria la posterior purificación del copolímero de injerto.

iii) El efecto de la temperatura es de poca importancia en la formación del radical por lo que se puede controlar la etapa de propagación.

iv) El efecto de aditivos, disolventes, etc. puede ser controlado usando un amplio rango de dosis de radiación y temperatura.

v) La polimerización puede llevarse a cabo en fase sólida.

vi) Fácil preparación cuando se compara con los métodos químicos convencionales.

vii) Aplicabilidad para una gran mayoría de combinación de polímeros debida a que la absorción de energía por parte de la materia no es selectiva.

viii) Mayor eficiencia de la transferencia de energía proveniente de la radiación cuando se compara con los métodos químicos que requieren calentamiento.

\subsubsection{Injerto por método directo}

En éste método, el polímero y el monómero (en fase gaseosa ó líquida) son irradiados simultáneamente [10-17]. La irradiación permite la formación de sitios activos en la matriz polimérica y en el monómero (Figura 11.7). La dosis e intensidad de dosis son parámetros muy importantes en este método. Una de las desventajas que presenta este método es la considerable formación de homopolímero, generalmente esto sucede cuando se emplea una proporción mayor en volumen de monómero con respecto al disolvente [1] o si el rendimiento de radicales provenientes del monómero es considerablemente mayor que el rendimiento de radicales provenientes de la matriz polimérica. Además, el injerto puede predominar si la matriz polimérica es tratada antes de la irradiación como por ejemplo hinchándola en una solución del monómero, en un disolvente sensible a la radiación o bien por la adición de ciertos compuestos como ácidos (ácido sulfúrico), sales inorgánicas $\left(\mathrm{LiClO}_{4}, \mathrm{LiNO}_{3}\right)$, etc. [18-21] 


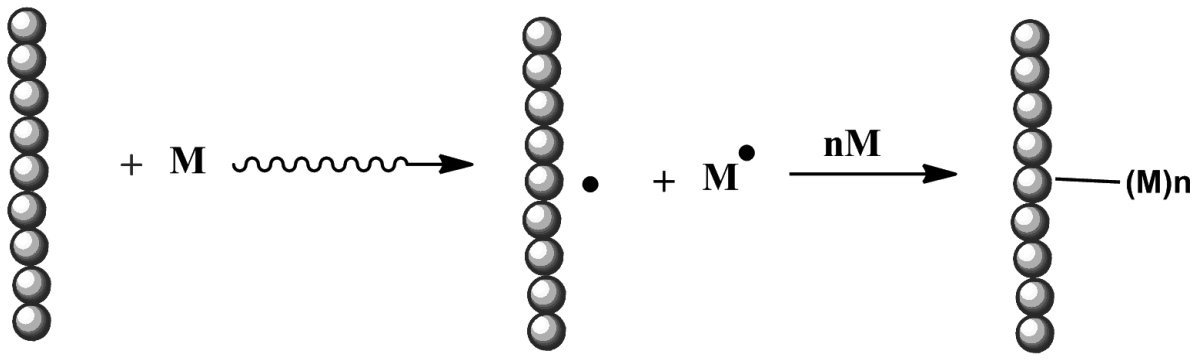

Figura 11.7. Proceso de injerto llevado a cabo por el método directo.

\subsubsection{Método de pre-irradiación oxidativa}

Alternativamente el injerto puede ser conseguido irradiando inicialmente la matriz polimérica en presencia de aire para formar hidroperóxidos y peróxidos, los cuales posteriormente con calentamiento forman radicales libres que comiencen el proceso de injerto (Figura 11.8). Una ventaja de este método es la posibilidad de almacenar el polímero irradiado a baja temperatura por un tiempo considerable. Además se reduce considerablemente la formación de homopolímero [22-28]. Una desventaja es la posible degradación del polímero.

\subsubsection{Método de pre-irradiación}

En este método la matriz polimérica es irradiada en ausencia de aire o en atmósfera de un gas inerte para formar sitios activos [29]. Posteriormente, la matriz polimérica irradiada se pone en contacto con el monómero en fase gaseosa o líquida (Figura 11.9). Una de las ventajas de este método es la poca formación de homopolímero. Se aconseja para este método llevar a cabo la irradiación y la difusión del monómero a bajas temperaturas para prevenir la recombinación de radicales. Una de las desventajas de este método es que el porcentaje de injerto suele ser muy bajo. 


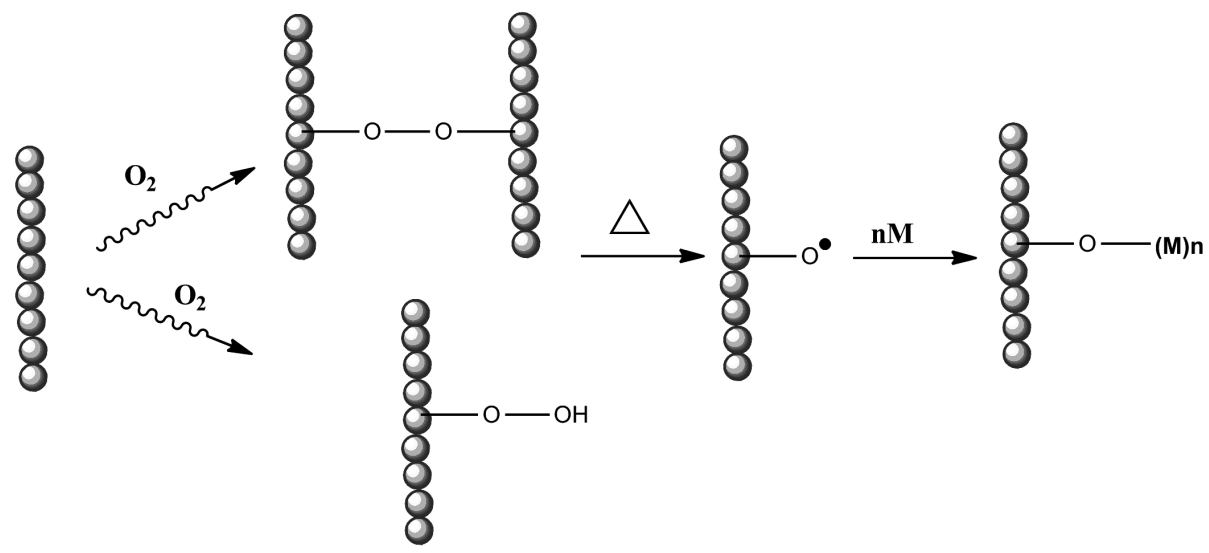

Figura 11.8. Proceso de injerto llevado a cabo por el método de pre-irradiación oxidativa.

\subsubsection{Irradiación directa en presencia de vapor}

En este método se emplea una ampolleta diseñada de tal forma que el monómero no esté en contacto con la película que se desee injertar, se elimina el oxígeno presente en el sistema; en un dispositivo de plomo se coloca la ampolleta cubriendo (blindando) únicamente el monómero, permitiendo que la radiación ionizante incida sobre la película, la cual estará en contacto con los vapores del monómero con lo que se iniciará el injerto. El mecanismo de injerto es igual al del método irradiación directa [30].

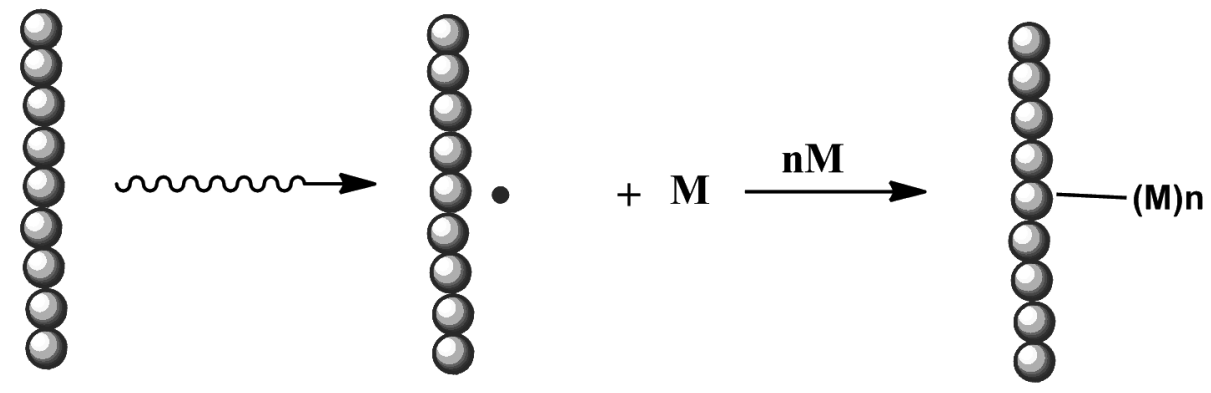

Figura 11.9. Proceso de injerto llevado a cabo por el método de pre-irradiación. 


\subsubsection{Otras técnicas de injerto}

\subsubsection{Injerto por activación química}

El injerto por activación química puede proceder mediante un mecanismo de radicales libres o un mecanismo iónico. En este método el papel del iniciador es muy importante tanto que determina el mecanismo del proceso de injerto. En el mecanismo por radicales libres, éstos son generados de iniciadores y transferidos a la matriz polimérica para que reaccionen con el monómero y obtener el copolímero de injerto. Entre los iniciadores empleados se pueden encontrar peróxido de benzoilo (BPO), azoisobutironitrilo (AIBN), persulfatos, iones metálicos tales como $\mathrm{Ce}^{4+}, \mathrm{Mn}^{3+}$ etc. La generación de radicales libres puede darse a través de una reacción de óxido-reducción [31], por oxidación directa de la cadena polimérica en presencia de ciertos metales [32], usando metales quelatados [33]. Una de las limitaciones de esta técnica es que se debe seleccionar un apropiado proceso oxidativo, por ejemplo, un sistema con alto potencial redox podría reaccionar con los monómeros incrementando la homopolimerización.

\subsubsection{Injerto fotoquímico}

Cuando un cromóforo que forma parte de una macromolécula absorbe luz se excita, lo cual puede resultar en una disociación de enlace y generar radicales libres causando el proceso de injerto. Si la absorción de luz no permite la formación de radicales libres a través de la ruptura de enlaces, este proceso puede ser promovido por la adición de fotosensibilizadores como, por ejemplo, ésteres de benzoina, benzofenona, iones metálicos de $\mathrm{UO}_{22}{ }^{+}$etc. Esto significa que el proceso de injerto inducido fotoquímicamente puede proceder mediante dos rutas: con o sin un fotosensibilizador [34-40]. El mecanismo sin fotosensibilizador involucra la generación de radicales libres sobre la matriz polimérica, los cuales reaccionan con el monómero para formar el copolímero de 
injerto. Por otro lado, en el mecanismo con fotosensibilizador, éste forma los radicales libres que pueden abstraer átomos de hidrógeno de la matriz polimérica produciendo sitios reactivos que son requeridos para que el proceso de injerto sea llevado a cabo.

Entre las características de esta técnica se encuentran: i) el injerto puede proceder a temperatura ambiente obteniendo altas conversiones de monómero, ii) el injerto puede ser controlado sobre la superficie polimérica sin afectar las propiedades en masa del polímero a injertar. Entre las desventajas de este método se puede mencionar que el injerto ocurre principalmente sobre la superficie y el polímero puede degradarse si se sobrepasa la dosis óptima.

\subsubsection{Injerto inducido por plasma}

En años recientes, la copolimerización de injerto inducida por la técnica de plasma ha recibido gran interés. Los principales procesos en plasmas son la excitación de electrones, la ionización y disociación del polímero o monómero. Es decir, los electrones acelerados del plasma tienen la suficiente energía para inducir el rompimiento de enlaces químicos en la matriz polimérica, generando radicales libres los cuales pueden subsecuentemente iniciar el proceso de injerto. Esencialmente, los radicales formados en las cadenas poliméricas pueden inicar reacciones similares a aquellas generadas con radiación de alta energía (por ejemplo, radiación gamma). La formación de radicales libres sobre la superficie del polímero predomina sobre su ionización. Debido a que los cambios sobre la matriz polimérica están confinados solamente a una profundidad de unos cuantos nanómetros sobre la superficie, las propiedades de la matriz polimérica (grado de polimerización y cristalinidad) no se ven muy influenciados en comparación con la radiación gamma. Entre las características de este tipo de injerto se encuentran: i) El injerto sobre la superficie es más favorecido, ii) el injerto puedo ser llevado a cabo sin añadir un fotosensibilizador, iii) los polímeros son primeramente expuestos al plasma para crear los radicales libres 
en la superficie y entonces puestos en contacto con el monómero, iv) diferentes tipos de plasma pueden ser empleados (plasma de argón, oxígeno) [41, 42]. Entre las limitaciones de esta técnica se encuentra su costo y que el injerto se restringe a la superficie del material a modificar.

\subsubsection{Injerto enzimático}

El principio involucrado en este método es que una enzima inicia la reacción de injerto. Por ejemplo la tirosinasa es capaz de convertir fenol a una o-quinona, la cual subsecuentemente será capaz de llevar a cabo el proceso de injerto [43, 44]. El uso de enzimas en reacciones de injerto ha sido extensamente estudiado en mayor proporción en biopolímeros que para polímeros sintéticos. [45, 46]. Entre las principales características de este tipo de injerto se encuentran: i) el injerto ocurre en condiciones suaves de reacción y usualmente no se requiere el uso de materiales peligrosos, ii) la etapa de iniciación requiere de la enzima para formar los radicales libres, iii) la selectividad de la enzima hace que la reacción sea simple y ofrece un mejor control sobre la estructura macromolecular. Sin embargo, esta técnica presenta varias limitaciones entre las cuales se encuentra su costo y que no puede ser llevada a cabo a altas temperaturas.

\subsection{Interacción de la radiación con la materia}

La materia está constituida por átomos, y la radiación ionizante interactúa con los electrones orbitales de los mismos con una probabilidad de ocurrencia que depende del tipo y energía de la radiación, así como también de la naturaleza del material. En todos los casos los resultados de la interacción de la radiación con la materia son la excitación y/o la ionización de los átomos del medio.

Entre los tipos de radiación ionizante utilizados se encuentran las partículas alfa, las partículas beta y las radiaciones electromagnéticas (radiación gamma). 
Los efectos de la radiación gamma o electromagnética con la materia que prevalecen para energías de algunas decenas de kilo electron voltios (keV) y aproximadamente 10 mega electronvoltios (MeV) son tres y el predominio de cada uno de ellos depende de la energía de radiación. Estos son: el efecto fotoeléctrico, efecto Compton y la producción de pares. Los dos primeros involucran interacciones con electrones orbitales de los átomos del absorbente. La producción de pares se manifiesta para energías superiores a $1.02 \mathrm{MeV}$. La interacción de los fotones con electrones origina el efecto fotoeléctrico y el efecto Compton mientras que la interacción con los núcleos origina la formación de pares.

En los átomos radiactivos, los núcleos atómicos emiten partículas subnucleares, o radiación electromagnética característica, sin masa ni carga, teniendo lugar un intercambio de energía al mismo tiempo.

\subsubsection{Radiación Gamma $(\gamma)$}

La radiación gamma $(\gamma)$ es producida generalmente por elementos radioactivos. Debido a las altas energías que poseen, constituyen un tipo de radiación ionizante capaz de penetrar en la materia más profundamente que la radiación alfa o beta provocando la formación de partículas cargadas eléctricamente llamadas iones. Los rayos $\gamma$ son radiaciones electromagnéticas, similares a los rayos $\mathrm{X}$, la luz u ondas de radio, pero con mucho menor longitud de onda y en consecuencia mucho mayor energía. Los rayos $\gamma$ al igual que los rayos $\mathrm{X}$, tienen energías bien definidas ya que son producidos por la transición entre niveles de energía del átomo. Sin embargo, los rayos $\gamma$ son emitidos por el núcleo mientras que los rayos $\mathrm{X}$ resultan de las transiciones de energía de los electrones fuera del núcleo u orbitales. La energía de este tipo de radiación se mide en megaelectronvoltios (MeV). Un Mev corresponde a fotones gamma de longitudes de onda inferiores a $10^{-11}$ $\mathrm{m}$ o frecuencias superiores a $10^{19} \operatorname{Hertz}(\mathrm{Hz})$. Los rayos $\gamma$ se producen con la desintegración de isótopos radiactivos como el ${ }^{60} \mathrm{Co}$. 


\subsubsection{Partículas Beta $(\beta)$}

Son partículas de masa despreciable (su masa es la del electrón o sea aproximadamente $1 / 1836$ de aquella del protón y 1/1834 de aquella del neutrón) y presentan carga negativa o positiva. Las partículas $\beta^{-}$o negatrón es un electrón emitido por el núcleo, el cual aumenta en 1 unidad su carga positiva, al transformar un neutrón en protón y un negatrón. Por tanto, el número atómico aumenta 1 unidad, el átomo se convierte en el elemento situado un lugar a la derecha en la tabla periódica y su número de masa permanece sensiblemente el mismo. La partícula, $\beta^{+}$o positrón, es emitida cuando un protón se transforma en neutrón y una partícula de la misma masa que el electrón pero con carga positiva es emitida por el núcleo. El número atómico decrece una unidad y el elemento se corre un lugar a la izquierda en la tabla periódica.

Las partículas $\beta$ de origen nuclear, tienen velocidades que pueden llegar a ser cercanas a la velocidad de la luz. Pese a ello sus energías son mayores, en general, a las de las partículas $\alpha$, ya que estas últimas en su mayoría no alcanzan los $4 \mathrm{MeV}$. Las partículas $\beta$ son mucho más penetrantes. Para tener una idea comparativa, una partícula $\alpha$ de $3 \mathrm{MeV}$ tiene un alcance en aire de 2.8 centímetros y produce alrededor de 4000 pares iónicos por milímetro de recorrido, mientras que una partícula $\beta$ de igual energía tiene un alcance en aire de más de 100 centímetros, y sólo produce 4 pares iónicos por milímetro.

\subsubsection{Partículas Alfa $(\alpha)$}

Las partículas $\alpha$ son núcleos de helio compuesto por dos neutrones y dos protones. Las partículas $\alpha$ emitidas por los núcleos atómicos, con energías comprendidas entre los 3 y los $9 \mathrm{MeV}$, se absorben fácilmente en la materia. Una hoja de papel o unos pocos centímetros de aire bastan para absorber totalmente partículas $\alpha$ producidas en reacciones nucleares. La pérdida de energía de estas partículas en el medio absorbente se debe principalmente a la ionización y excitación. 


\subsubsection{Unidades de interacción de la radiación ionizante con la materia}

Para entender el concepto de interacción de la radiación con la materia es necesario definir algunas unidades; el primero es el roentgen " $R$ " que representa la generación de una unidad electrostática de carga en un centímetro cúbico de aire en condiciones normales de presión y temperatura. Esta unidad entiende a todo lo referente a exposición pero al estudiar la materia lo importante es la dosis absorbida; este concepto se explica mediante el "rad" que equivale a una transferencia de 100 ergios por cada gramo de materia. Actualmente en el Sistema Internacional de Unidades la dosis absorbida se expresa en gray (Gy) que se define como la cantidad de energía absorbida por cada kg de materia y se mide en Joules $\mathrm{kg}^{-1}$.

\subsection{Cinética del proceso de injerto inducido por radiación}

Los injertos inducidos por radiación se pueden producir por mecanismos vía radicales libres, iónico y mezcla de ambos. En un mecanismo vía radicales libres, el injerto dependerá de la difusión del monómero en el polímero, de la reactividad de los radicales producidos y de la polimerización del monómero, principalmente.

\subsubsection{Cinética de polimerización e injerto por radiación vía radicales libres}

En el primer paso, la irradiación de monómeros o polímeros da como resultado la ionización de moléculas:

$\mathrm{M}$ o $\mathrm{P} \leadsto \mathrm{M}^{+} \mathrm{o} \mathrm{P}^{+}+\mathrm{e}^{-}$ Ecuación (11.1)

Donde $\mathrm{M}=$ monómero y $\mathrm{P}=$ matriz polimérica.

En un segundo paso, el electrón es capturado por un ión $\mathrm{M}^{+} \mathrm{O} \mathrm{P}^{+}$ produciendo moléculas excitadas 


$$
\mathrm{M}^{+} \circ \mathrm{P}^{+}+\mathrm{e}^{-} \longrightarrow \mathrm{M}^{*} \mathrm{o} \quad \mathrm{P}^{*}
$$

Y finalmente en un tercer paso, la molécula con energía de excitación de 8 a 15 eV se descompone en radicales ( $R$ ):

$$
\mathrm{M}^{*} \mathrm{o} \mathrm{P}^{*} \longrightarrow 2 \mathrm{R}^{*} \quad \text { Ecuación (11.3) }
$$

Análogamente, las moléculas pueden quedar excitadas inicialmente formando $\mathrm{M}^{* *}$ o $\mathrm{P}^{* *}$ cuando la energía de irradiación es pequeña para su ionización:

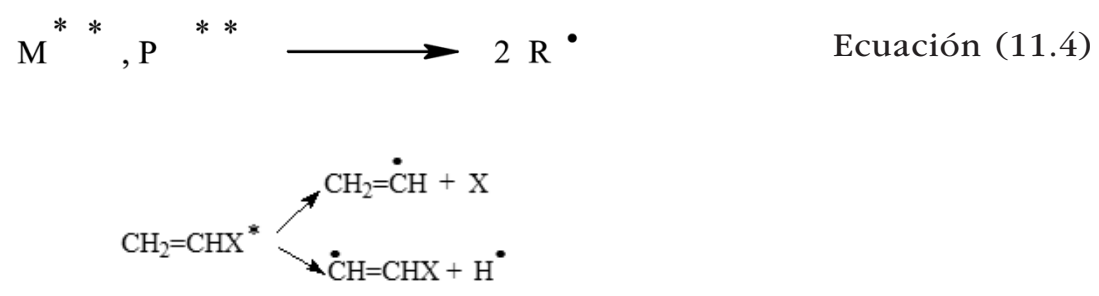

Ecuación (11.5)

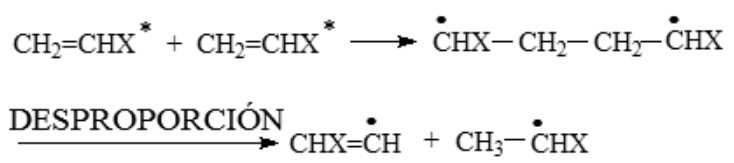

En la irradiación de polímeros se pueden formar varios radicales. Los macro-radicales formados en una matriz polimérica con sitios activos promueven la iniciación del injerto. La velocidad de iniciación de polimerización (Vi) se expresa mediante la ecuación 11.6:

$$
V i=\frac{G_{R} I}{100 N_{A}}[=] \frac{\mathrm{mol}}{1 \mathrm{sec}} \quad \mathrm{I}[=] \mathrm{eV} / 1 \mathrm{sec}
$$

Ecuación (11.6)

Donde: $\quad G_{R}=\frac{[M] N_{A} 100}{\text { I t }}$ 
$\mathrm{I}=$ Intensidad de irradiación, $[\mathrm{M}]=$ concentración de monómero, $\mathrm{N}_{\mathrm{A}}$ = número de Avogadro, $\mathrm{t}=$ tiempo, $\mathrm{G}_{\mathrm{R}}=$ rendimiento radioquímico .

La ecuación 11.7 expresa la velocidad de propagación (Vp) mediante el siguiente esquema de reacción:

$$
\mathrm{Vp}=\mathrm{kp}\left[\mathrm{RM}^{\bullet}\right][\mathrm{M}]
$$

La ecuación 11.8 expresa la velocidad de terminación (Vt) mediante el mecanismo de radicales libres.

$$
\mathrm{Vt}=\mathrm{kt}[\mathrm{RM}]^{2 *}
$$

Por último, la velocidad total de polimerización (V) se expresa:

$$
\mathrm{V}=-\mathrm{d}[\mathrm{M}] / \mathrm{dt}=\mathrm{kp}\left[\mathrm{RM}^{\bullet}\right][\mathrm{M}] \quad \text { Ecuación (11.9) }
$$

En el estado estacionario la concentración de radicales en el sistema está determinada por las expresiones mostradas en la ecuación 11.10.

$$
\begin{aligned}
\mathrm{R}^{\bullet}+\mathrm{M} & \longrightarrow \mathrm{RM}^{\bullet} \\
\mathrm{RM}^{\bullet}+\mathrm{M} & \longrightarrow \mathrm{RM}_{1}^{\bullet}
\end{aligned}
$$

La velocidad de polimerización mediante radiación y radicales libres es proporcional a I ${ }^{0.5}$ y $[\mathrm{M}]$. A continuación se muestra el proceso de polimerización por radicales libres iniciada con radiación:

$$
\begin{array}{cc}
\mathrm{R}^{*}+\mathrm{M} \longrightarrow \mathrm{RM}^{*} & \begin{array}{l}
\text { RADICALES EN LA } \\
\text { MATRIZ POLIMÉRICA }
\end{array} \\
\mathrm{RM}^{*}+\mathrm{M} \longrightarrow \mathrm{RM}_{1}^{*} & \text { INICIACIÓN } \\
\mathrm{RM}^{*}+\mathrm{RM}^{*} & \text { PROPAGACIÓN } \\
\text { TERMINACIÓN }
\end{array}
$$


Utilizando el principio del estado estacionario y considerando las ecuaciones de cinética vistas anteriormente, la velocidad de injerto se expresa en la ecuación 11.11:

$$
\begin{aligned}
& \text { Donde } \quad \mathrm{Vi}=\mathrm{Vt} \\
& \mathrm{ki}\left[\mathrm{R}^{\circ}\right][\mathrm{M}]=\mathrm{kt}\left[\mathrm{RM}^{\circ}\right]^{2} \\
& {\left[\mathrm{RM}^{\circ}\right]=\frac{\mathrm{ki}^{0.5}[\mathrm{R}]^{0.5}[\mathrm{M}]^{0.5}}{\mathrm{kt}^{0.5}}}
\end{aligned}
$$

Velocidad de Injerto

$$
\mathrm{V} \text { injerto }=\frac{\mathrm{ki}^{0.5}{\mathrm{kp}\left[\mathrm{R}^{\cdot}\right]^{0.5}[\mathrm{M}]}_{\mathrm{kt}^{0.5}}^{1.5}}{{ }^{0.5}}
$$

Nota: $\mathrm{Si}\left[\mathrm{R}^{\bullet}\right]$ es proporcional a $\mathrm{I}$, el número de radicales producidos en la reacción de injerto será proporcional a $\mathrm{I}^{0.5}$ y $[\mathrm{M}]^{1.5}$ lo cual corresponde a numerosos datos experimentales, la masa molecular es proporcional a $\mathrm{I}^{-0.5}$.

Por otro lado, el índice de polimerización (Pn) está determinado por la relación de velocidad de polimerización e iniciación (ecuación 11.12).

$$
\mathrm{P}_{\mathrm{n}}=\frac{\mathrm{V}}{\mathrm{Vi}}=\frac{\mathrm{ki}^{0.5} \mathrm{kp} \mathrm{I}^{0.5}}{\mathrm{kt}^{0.5} \mathrm{ki} \mathrm{I}}[\mathrm{M}]=\frac{\mathrm{ki}^{-0.5} \mathrm{kp} \mathrm{I}^{-0.5}}{\mathrm{kt}^{0.5}}[\mathrm{M}]
$$

\subsection{Efecto de varios factores en polimerización y velocidad de injerto}

La velocidad de polimerización depende de la naturaleza del monómero; por ejemplo el acetato de vinilo polimeriza 100 veces más rápido que el estireno así para tener mayor rendimiento $G_{R^{\prime}(P)}>G_{R^{\prime}(M)}$ (el rendimiento radioquímico de formación de radicales del polímero deberá ser mayor que el del monómero). 
Hasta el momento no hay ninguna regla que nos indique cuales son las variables o condiciones adecuadas para obtener un copolímero de injerto con el rendimiento deseado o con las mejores propiedades mecánicas.

\subsubsection{Efecto de la intensidad de radiación}

A altas intensidades (razones de dosis) la polimerización vía radicales decrece debido a la recombinación de los radicales producidos [47, 48]. La velocidad de radicales en injertos en muchos casos es proporcional a la raíz cuadrada de la intensidad de radiación $\mathrm{V} \sim \mathrm{I}^{0.5}$.

\subsubsection{Efecto de la dosis}

A altas dosis de radiación la velocidad de polimerización se incrementa debido a un proceso de autoaceleración y se incrementa el porcentaje de injerto [49], el cual también depende de la naturaleza del monómero y de la intensidad de radiación. La auto-aceleración es causada por un incremento de viscosidad en el sistema ocasionado por una posible disminución de la velocidad de terminación sin afectar la velocidad de propagación de la cadena (efecto gel). De esta forma, se presenta la formación de nuevos radicales no sólo en el monómero sino también en las cadenas poliméricas en crecimiento que pueden iniciar una polimerización (auto-catálisis).

\subsubsection{Efecto de la temperatura}

La velocidad de polimerización o de injerto se incrementa con la temperatura; en este caso también dependerá de la naturaleza del monómero ya que al llegar a una cierta temperatura combinada con factores como la difusión, concentración, dosis etc. puede ocurrir la homopolimerización $[50,51]$. Como se mencionó anteriormente, a mayor temperatura 
se incrementa el porcentaje de injerto hasta llegar a una temperatura óptima que no disuelva al copolímero de injerto o a la matriz a injertar.

\subsubsection{Efecto de la concentración de monómero}

En este caso, la concentración de monómero utilizada dependerá del método empleado (pre-irradiación o directo). Por ejemplo, si se escoge el método directo para llevar a cabo el injerto, no es conveniente manejar concentraciones de monómero superiores a $80 \%$ porque hay una mayor tendencia a formar homopolímero que hacia el proceso de injerto. Por otro lado, si se utiliza el método de pre-irradiación debemos utilizar concentraciones de monómero superiores al 50\% ya que a concentraciones más pequeñas el injerto será muy pobre porque hay menor cantidad de radícales que copolimerizará con la matriz polimérica [52-55].

\subsubsection{Efecto del disolvente}

En el caso de la irradiación del sistema: polímero-monómero-disolvente (método directo), es importante que la estructura química del monómero y el disolvente sea similar así como también para el método de preirradiación [56-58]. En ambos casos es muy importante tomar en cuenta que el disolvente empleado debe ser soluble en el monómero, pero nodisolvente de la matriz polimérica a injertar así como del copolímero de injerto, dicho disolvente debe ser capaz de hinchar el polímero a injertar. En el caso más simple, cuando no existe transferencia de energía de excitación entre los componentes. La velocidad de iniciación es:

$$
\mathrm{Vi}=[\mathrm{G}(\mathrm{R}) \mathrm{m} \mathrm{fm}+\mathrm{G}(\mathrm{R}) \mathrm{s} \text { fs }] \times\left[\mathrm{I} / 100 \mathrm{~N}_{\mathrm{A}}\right] \quad \text { Ecuación (11.13) }
$$

Donde $G(R) m$ y $G(R)$ s son los rendimientos de radicales del monómero

y del disolvente, fm y fs son las fracciones molares de los mismos, en este caso la velocidad de polimerización se expresa como: 
$\mathrm{V}=\mathrm{Kp} \mathrm{kt} \mathrm{t}^{0.5}[\mathrm{M}] \times\left\{[\mathrm{G}(\mathrm{R}) \mathrm{m} \mathrm{fm}+\mathrm{G}(\mathrm{R}) \mathrm{s} \text { fs }] \times\left[\mathrm{I} / 100 \mathrm{~N}_{\mathrm{A}}\right]\right\}^{0.5}$ Ecuación (11.14)

\subsubsection{Efecto del espesor de la matriz polimérica}

En el proceso de injerto de polímeros, el incremento depende de la difusión del monómero en la película. Existen varios trabajos en los cuales se estudia la cinética de injerto con respecto al espesor. El efecto de la difusión del monómero trae como resultado una reducción de la velocidad de injerto de películas gruesas comparadas con películas delgadas [59, 60].

\subsubsection{Sensibilidad a la radiación ionizante en el sistema polímero/monómero}

La radiación ionizante no es selectiva y tiene efecto sobre la matriz polimérica, monómero, disolvente y cualquier otra especie presente en el sistema. La sensibilidad a la radiación de una especie química es medida en términos de su valor de rendimiento radioquímico (G). El injerto es favorecido cuando el valor del rendimiento radioquímico de la matriz polimérica es mayor que el del monómero [61, 62].

\subsection{Técnicas de caracterización}

En la actualidad es muy importante la síntesis de nuevos materiales, para una posible aplicación en la industria; es aquí donde juega un papel importante la caracterización de un nuevo material, ya que existen técnicas complementarias con las cuales podemos saber los grupos químicos presentes, comportamiento térmico, grado de cristalinidad, resistencia mecánica, etc.

\subsubsection{Espectroscopia de infrarrojo (FT-IR)}

La radiación infrarroja en el rango de 10000-100 $\mathrm{cm}^{-1}$ es absorbida y convertida por una molécula a energía de vibración molecular. Esta 
absorción puede ser cuantificada y la longitud de onda de absorción depende de las masas relativas de los átomos, la fuerza de enlaces y la geometría de los átomos. Las intensidades de las bandas pueden ser expresadas en términos de transmitancia ( $\mathrm{T}$ ) o absorbancia (A). Existen dos tipos de vibración molecular: estiramiento y torsión. Un estiramiento es un movimiento a través del enlace tal que la distancia interatómica se incrementa o decrece mientras que una torsión consiste en un cambio en el ángulo entre enlaces con un átomo en común

La formación del copolímero de injerto se puede comprobar mediante espectroscopia de infrarrojo comparando los espectros de la matriz polimérica y el copolímero de injerto. El copolímero de injerto mostrará señales adicionales (estiramientos y torsiones) debidas a los átomos pertenecientes a las cadenas injertadas en la matriz polimérica [63, 64].

\subsubsection{Análisis termogravimétrico (TGA)}

Esta técnica permite observar la resistencia térmica de los copolímeros con respecto a la temperatura, es decir, en una gráfica de porcentaje de pérdida de peso contra temperatura se puede determinar la temperatura a la cual se descompone el copolímero [65, 66]. El TGA mide la variación de masa en un compuesto en función de la temperatura. Entre los cambios térmicos que se acompañan de un cambio de masa se encuentran la descomposición, la sublimación, la reducción, la desorción, la absorción y la vaporización. Las mediciones son normalmente llevadas a cabo en aire o en atmósfera inerte de helio, argón ó nitrógeno y el peso es registrado como una función de la temperatura.

\subsubsection{Calorimetría diferencial de barrido (DSC)}

Cuando un material pasa por un cambio de estado físico, por ejemplo, una fusión o una transición cristalina de una forma a otra, o cuando reacciona químicamente, tiene lugar una absorción o un desprendimiento 
de calor. Muchos de estos procesos pueden ser iniciados simplemente aumentando la temperatura del material. Los calorímetros diferenciales de barrido están diseñados para determinar las entalpías de estos procesos, midiendo el flujo calorífico diferencial requerido para mantener una muestra del material, y una referencia inerte a la misma temperatura. El método es uno de los más importantes en la determinación de las mesofases de un cristal líquido.

En polímeros que presentan un cierto grado de cristalinidad, el estudio de DSC es empleado para determinar el calor de fusión y consecuentemente los cambios en el grado de cristalinidad de la matriz polimérica y en el copolímero de injerto. De los experimentos de DSC se puede obtener información sobre la temperatura de transición vítrea (Tg) y temperatura de fusión (Tm) [67-70]. La calorimetría diferencial de barrido (DSC) es una técnica muy empleada para la determinación de la Tg de sistemas poliméricos debido a la mínima cantidad de polímero requerido y lo confiable de sus mediciones. La Tg se puede entender de forma bastante simple cuando se entiende que en esa temperatura el polímero deja de ser rígido y comienza a ser blando. Se entiende que es un punto intermedio de temperatura entre el estado fundido y el estado rígido del polímero. A temperaturas superiores de la $\mathrm{Tg}$ los enlaces de las moléculas son mucho más débiles que el movimiento térmico de las mismas, por ello el polímero adquiere cierta elasticidad y capacidad de deformación plástica. Este cambio no ocurre repentinamente, sino que tiene lugar a través de un rango de temperaturas. Esto hace que resulte un poco complicado escoger una Tg discreta pero generalmente se determina como $\mathrm{Tg}$ el punto medio de ese rango de temperaturas. Por otro lado, cuando se alcanza la temperatura de fusión del polímero los cristales poliméricos comenzarán a separarse, es decir, se funden. Las cadenas abandonan sus arreglos ordenados y comienzan a moverse libremente. Cuando los cristales poliméricos funden deben absorber calor para poder hacerlo, es decir, la fusión es una transición endotérmica. La fusión sólo se presenta en aquellos polímeros capaces de formar cristales mientras que los polímeros completamente amorfos no la presentan. 


\subsubsection{Hinchamiento}

Algunos polímeros (tales como los hidrogeles) son capaces de captar grandes cantidades de agua, manteniendo su estructura tridimensional, en cantidades que dependen de la hidrofilicidad de los polímeros constituyentes. Este proceso además es reversible y dependiente de las condiciones ambientales. El mecanismo por el que los polímeros son capaces de absorber un cierto volumen de solución acuosa no es solamente físico, sino que depende de la naturaleza química del polímero. Por un lado, la diferencia entre la concentración de iones, el hidrogel hinchado y la solución externa produce una presión osmótica, que sólo puede reducirse a través de la dilución de carga, es decir, por el hinchamiento del gel, y por la densidad de carga neta entre las cadenas que genera repulsiones electrostáticas que tienden a expandir el gel, lo que contribuye al hinchamiento. La técnica de hinchamiento se utiliza con frecuencia para determinar el parámetro de solubilidad de los polímeros. Se basa en evaluar el hinchamiento del polímero en una serie de disolventes de parámetros de solubilidad conocidos. Se asume que se alcanzará el máximo hinchamiento cuando el parámetro de solubilidad del disolvente sea igual al del polímero. El hinchamiento puede definirse en términos de velocidad y de equilibrio de máxima absorción y define la capacidad de penetración de las moléculas de disolvente en el polímero, dependiendo de la naturaleza del disolvente [71-73].

\subsubsection{Difracción de rayos-X}

Esta técnica nos ayuda a determinar el ordenamiento en la estructura de un compuesto, es decir; si el compuesto tiene un alto ordenamiento presentará una estructura cristalina; si por el contrario, no hay ordenamiento el compuesto presenta una estructura amorfa. Las gráficas representativas de esta técnica se llaman difractogramas. Cuando se presentan máximos orientados se considera que el compuesto es cristalino y cuando no hay máximos, sino una zona ancha de dispersión el compuesto es amorfo 
[74-77]. Cuando se utiliza esta técnica con una platina de calentamiento controlado, es muy útil en la determinación de un cristal líquido polimérico.

\subsection{6. Ángulo de contacto}

Es una medida cuantitativa de la capacidad para humedecerse de un sólido por un líquido. Para el análisis de superficie de películas poliméricas se define geométricamente como el ángulo formado por un líquido en el límite de tres fases donde un líquido (usualmente agua), un gas (aire) y un sólido (copolímero) se intersectan. Cuanto más grande es el ángulo tanto menor es la interacción que existe entre el líquido (agua) y el sólido (polímero). Si el líquido (agua) es fuertemente atraído hacia la superficie de un sólido hidrofílico, la gota se extenderá completamente sobre la superficie y los valores de ángulo serán cercanos a $0^{\circ}$ [78, 79]. Los sólidos menos fuertemente hidrofilicos tendrán valores de ángulo de contacto menores de $90^{\circ}$ mientras que para superficies hidrofóbicas el ángulo de contacto será mayor de $90^{\circ}$ [80]. Incluso para superficies altamente hidrofóbicas se pueden observar ángulos de contacto mayores de $150^{\circ}$.

Mediante la medición del ángulo de contacto no solo se pueden determinar las propiedades hidrofílicas/hidrofóbicas de la superficie de un material sino que además el ángulo de contacto es un parámetro de interés en la flotación para la obtención de minerales, en la determinación de la tensión superficial de sólidos, análisis de superficies de materiales para uso biomédico (por ejemplo para evaluar la limpieza de estos materiales) y en el estudio de adhesión/ repelencia sobre superficies poliméricas. Sin embargo, varios factores pueden afectar los resultados cuando se lleva a cabo la medición del ángulo de contacto, tales como, la contaminación, humedad y rugosidad de la superficie.

\subsubsection{Microscopio electrónico de barrido}

Con esta técnica podemos conocer la morfología de la superficie de la película injertada ya que esta técnica hace un barrido de la superficie. 
La preparación de las muestras requiere un recubrimiento de oro o de algún otro metal tal como plata o platino; además con la ayuda del microscopio podemos saber el tamaño aproximado de los poros en caso de polímeros reticulados y observar el grado de homogeneidad de la cadena injertada [81, 82].

\subsubsection{Injerto de diferentes monómeros derivados de acrilatos}

Los injertos pueden ser con un solo monómero o binario en presencia de dos monómeros; en este último caso el injerto puede realizarse en un paso (los dos monómeros y la matríz polimérica) o en pasos sucesivos injertando primero uno de ellos y a continuación el segundo.

El injerto inducido mediante radiación ionizante de $\mathrm{N}$-isopropilacrilamida (NIPAAm) sobre PP-g-AAc, PP-g-DMAAm, PP-g-DMAEMA, y PTFE-g-AAc; 4VP sobre PP-g-DMAEMA fueron investigados en función de la dosis de irradiación. El efecto de la dosis de irradiación sobre el porcentaje de injerto (segundo paso) fue evaluado llevando a cabo polimerizaciones de injerto con diferentes monómeros (NIPAAm y 4VP) y matrices poliméricas (PP y PTFE) usando los métodos directo y de pre-irradiación. El NIPAAm fue injertado en diferentes copolímeros de injerto (ver Figura 11.10): PP-g-DMAEMA, $100 \%$ de injerto (๑); PP-g-AAc, $50 \%$

de injerto (ם); PP-g-DMAAm, $35 \%$ de injerto ( $\boldsymbol{\Delta}$ ); PTFE-g-AAc, $100 \%$ de injerto $(\diamond)$.

Por otro lado, la $4 \mathrm{VP}$ fue injertada en PP-g-DMAEMA por método directo en diclorometano, obteniendo un $40 \%$ de injerto. Se espera que el número de sitios activos para llevar a cabo el injerto incremente con el aumento en la dosis de irradiación. Los resultados muestran que la eficiencia de injerto es una función de las matrices poliméricas así como de las condiciones de reacción. El valor más bajo de injerto fue obtenido para la combinación DMAEMA/NIPAAm en PP mientras que el más alto fue para DMAAm/NIPAAm en PP. El injerto de DMAAm en PP incrementa la hidrofilicidad del sistema (mayor hinchamiento en agua) y por lo tanto se incrementa el injerto del segundo monómero 
(NIPAAm). El injerto de 4VP en PP-g-DMAEMA (sintetizado por el método directo) tuvo un máximo de eficiencia de injerto de $95 \%$ a $30 \mathrm{kGy}$; el injerto llevado a cabo a valores superiores de esta dosis de irradiación permaneció casi constante. Es de importancia resaltar que este último sistema fue imposible de obtenerse por el método de pre-irradiación $[6,58,83-86]$.

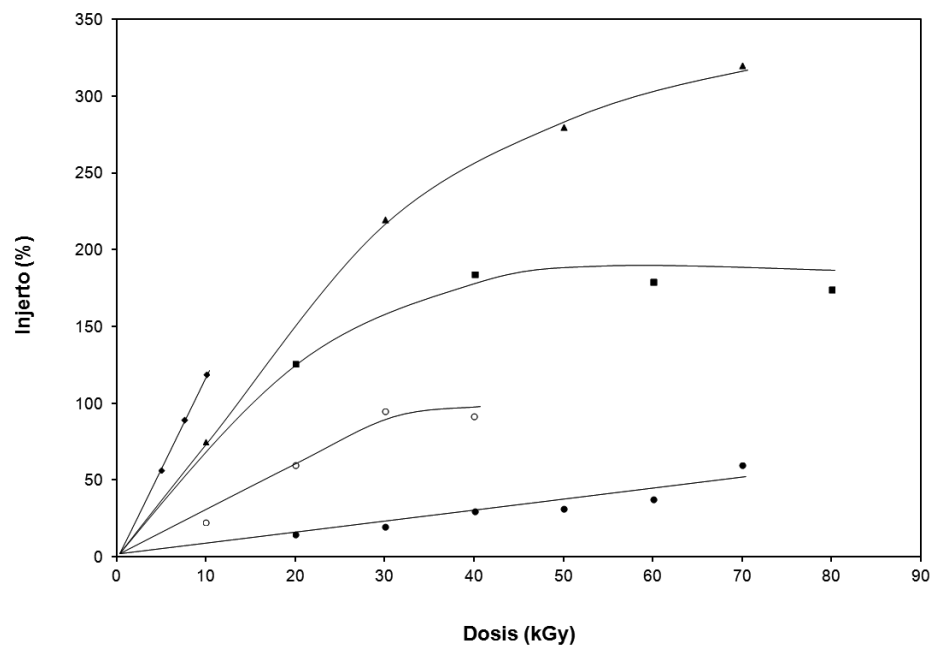

Figura 11.10. Injerto en función de la dosis, de NIPAAm injertado en diferentes matrices, por el método de pre-irradiación: (PP-g-DMAEMA 100\%) (•), (PP-g-AAc 50\%) (ロ), (PP-g-DMAAm 35\%) (A), (PTFE-g-AAc 100\%) (\). 4VP sobre (PP-gDMAEMA 40\%) (O) por el método de irradiación directa

\subsection{Agradecimientos}

Los autores agradecen a la M.L. Escamilla, M. Cruz y E. Palacios de ICN-UNAM por la asistencia técnica. Este trabajo fue apoyado por DGAPAUNAM IN200714, CONACYT-CNPq 174378, y "Red iberoamericana de nuevos materiales para el diseño de sistemas avanzados de liberación de fármacos en enfermedades de alto impacto socioeconómico" (RIMADEL), CYTED 211RT0423. 


\subsection{Referencias}

[1] A. Chapiro, Radiation Chemistry of Polymeric Systems, Wiley and Sons: New York, 1962.

[2] A. Chapiro, Radiat. Phys. Chem. 1977, 9, 55.

[3] G. Odian, M. Sobel, A. Rossi, R. Klein, J. Polym. Sci. 1961, 55, 663.

[4] T.R. Dargaville, G.A. George, D.J.T. Hill, A.K. Whittaker, Prog. Polym. Sci. 2003, 28, 1355.

[5] R. Mazzei, G. García, G. Massa, A. Filevich, Nucl. Instrum. Meth. B 2007, 255, 314.

[6] Y.S. Ramírez-Fuentes, E. Bucio, G. Burillo, Nucl. Instrum. Meth. B 2007, 265, 183.

[7] G. Moad, D.H. Solomon, The Chemistry of Radical Polymerization, Elsevier Ltd: Oxford, 2006.

[8] E. Rizzardo, J. Chiefari, B.Y.K. Chong, F. Ercole, J. Krstina, J. Jeffery, T.P.T. Le, R.T.A. Mayadunne, G.F. Meijs, C.L. Moad, G. Moad, S.H. Thang, Macromol. Symp.1999, 143, 291.

[9] G. Moad, E. Rizzardo, S.H. Thang, Aust. J. Chem. 2005, 58, 379.

[10] K. Abrol , G.N. Qazi, A.K. Ghosha, J. Biotechnol. 2007, 128, 838.

[11] K. Kumar, B.S. Kaith, R. Jindal, H. Mittal, J. Appl. Polym. Sci. 2012, 124, 4969.

[12] N.K. Goel, V.Kumar, M.S. Rao, Y.K. Bhardwaj, S. Sabharwal, Radiat. Phys. Chem. 2011, $80,1233$.

[13] A. El-Hag Ali, N.M. El-Sawy, E.S.A. Hegazy, A. Awadallah-F, Polym. Bull. 2011, 67, 1837.

[14] B. Singh, V. Sharma, A. Kumar, S. Kumar, Int. J. Biol. Macromol. 2009, 45, 338.

[15] H.H. Sokker, A.M.A. Ghaffar, Y.H. Gad, A. S. Aly, Carbohyd. Polym. 2009, 75, 222.

[16] T.B. Mostafa, J. Appl. Polym. Sci. 2009, 111, 11.

[17] G. González, G. Burillo, Radiat. Phys. Chem. 2010, 79, 870.

[18] J. Kunze and H.P. Fink, Macromol. Symp. 2005, 223, 175.

[19] E. Takacs, L. Wojnarovits, J. Borsa, J. Papp, P. Hargittai, L. Korecz, Nucl. Instrum. Meth. B $2005,236,259$.

[20] L. Andreozzi, V. Castelvetro, G. Ciardelli, L. Corsi, M. Faetti, E. Fatarella, F. Zulli, J. Colloid Interface Sci. 2005, 289, 455.

[21] A. Contreras-García, C. Alvarez-Lorenzo, A. Concheiro, E. Bucio, Radiat. Phys. Chem. 2010, 79, 615 .

[22] F. Muñoz-Muñoz, J.C. Ruiz, C. Alvarez-Lorenzo, A. Concheiro, E. Bucio, Radiat. Phys. Chem. 2012, 81, 531 .

[23] L. García-Uriostegui, G. Burillo, E. Bucio, Radiat. Phys. Chem. 2012, 81, 295.

[24] J.Y. Lee, HS. Wang, M.J. Han, G.C. Cha, S.H. Jung. S. Lee, J.Y. Jho, Macromol. Res. 2011, $19,1014$.

[25] S. Cetin, T. Tincer, J. Appl. Polym. Sci. 2008, 108, 414.

[26] S. Chawla, A.K. Ghosh, D.K. Avasthi, P. Kulriya, S. Ahmad, J. Appl. Polym. Sci. 2007, 105,3578 .

[27] Y. Kimura, M. Asanob, J. Chenb, Y. Maekawab, R. Katakaia, M. Yoshida, Radiat. Phys. Chem. 2008, 77, 864.

[28] F. Muñoz-Muñoz, J.C. Ruiz, C. Alvarez-Lorenzo, A. Concheiro, E. Bucio, Eur. Polym. J. 2009, 45, 1859.

[29] A. Bhattacharya, Prog. Polym. Sci. 2000, 25, 371. 
[30] E. Bucio, G. Cedillo, G. Burillo, T. Ogawa, Polymer Bull. 2001, 46, 115.

[31] J.A.G. Barros, G.J.M. Fechine, M.R. Alcantara, L.H. Catalani, Polymer 2006, 47, 8414.

[32] M.D. Kurkuri, J.R. Lee, J.H. Han, I. Lee, Smart Mater. Struc. 2006, 15, 417.

[33] B.N. Misra, J.K. Jassal, R. Dogra, J. Macromol. Sci. Chem. A 1981, 16, 1093.

[34] J.E. Kennedy, C.L. Higginbotham, Mat. Sci. Eng. C 2011, 31, 246.

[35] H. Zhao, Y. Feng, J. Guo, J. Appl. Polym. Sci. 2011, 119, 3717.

[36] C. Ignacio, I.A.S. Gomes, R.L. Oréfice, J. Appl. Polym. Sci. 2011, 121, 3501.

[37] M. Taniguchi, J. Pieracci, W.A. Samsonoff, G. Belfort, Chem. Mater. 2003, 15, 3805.

[38] G.S. Irwan, S.I. Kuroda, H. Kubota, T. Kondo, J. Appl. Polym. Sci. 2002, 83, 2454.

[39] D. He, M. Ulbricht, J. Mater. Chem. 2006, 16, 1860.

[40] T. Rohr, D.F. Ogletree, F. Svec, M.J. Frecht, Adv. Func. Mat. 2003, 13, 264.

[41] T. Kai, S. Yamaguchi, S. Nakao, Ind. Eng. Chem. Res. 2000, 39, 3284.

[42] A. Wenzel, H. Yanagishita, D. Kitamoto, A. Endo, K. Haraya, T. Nakane, N. Hanai, H. Matsuda, N. Koura, H. Kamusewitz, D. Paul, J. Membr. Sci. 2000, 179, 69.

[43] R. Jayakumar, M. Prabaharan, R.L. Reis, J.F. Mano, Carbohyd. Polym. 2005, 62, 142.

[44] S. Kobayashi, H. Hideyuk, Prog. Polym. Sci. 2003, 289, 1015.

[45] J. C. Zhao, Z.H. Xie, Z.A. Guo, G.J. Liang, J.L. Wang, Appl. Surf. Sci. 2004, 229, 124.

[46] J. Zhao, G. Fan, Z. Guo, Z. Xie, G. Liang, Polym. Bull. 2005, 55, 1.

[47] M.M. Nasef, E.A. Hegazy, Prog. Polym Sci. 2004, 29, 499.

[48] G.M. Iskander, L.E. Baker, D.E. Wiley, T.P. Davis, Polymer 1998, 39, 4165.

[49] E.S.A. Hegazy, H. Kamal, N. Maziad, A.M. Dessouki, Nucl. Instrum. Meth. B, 1999, $151,386$.

[50] R.J. Woods, A.K. Pikaev, Applied Radiation Chemistry: Radiation Processing, Wiley: New York, 1994

[51] L. Gubler, N. Prost, S.A. Gürsel, G.G. Scherer, Solid State Ionics, 2005, 176, 2849.

[52] M. Dole, Radiation Chemistry of Macromolecules, Academic Press: New York, 1973.

[53] Z. Xu, J. Wang, L. Shen, D. Men, Y. Xu, J. Membr. Sci. 2002, 196, 221.

[54] S.H. Choi, Y.C. Nho, Radiat. Phys. Chem. 2000, 58, 157.

[55] N. Walsby, M. Paronen, J. Juhanoja, F. Sundholm. J. Polym. Sci. A Chem. 2000, 38, 1512.

[56] B. Gupta, N. Anjum, A.P. Gupta, J. Appl. Polym. Sci. 2000, 77, 1401.

[57] F. Ranogajec, Radiat. Phys. Chem. 2007, 76, 1381.

[58] E. Bucio, G. Burillo, Radiat. Phys. Chem. 1996, 48, 805.

[59] F. Ranogajec, I. Dvornik, J. Dobo, Eur. Polym. J. 1970, 6, 1169.

[60] H.P. Brack, H.G. Bührer, L. Bonorand, G.G. Scherer, J. Mater. Chem. 2000, 10, 1975.

[61] F. Cardona, G.A. George, D.J.T. Hill, F. Rasoul, J. Maeji, Macromolecules 2002, 35, 355.

[62] H. Sakurai, M. Shiotani, H. Yahiro, Radiat. Phys. Chem. 1999, 56, 309.

[63] C. Qiu, F. Xu, Q.T. Nguyen, Z. Ping, J. Membr. Sci. 2005, 255, 107.

[64] M. Kallrot, U. Edlund, A.C. Albertsson, Biomaterials 2006, 27, 1788.

[65] M.E. Brown, Introduction to Thermal Analysis: Techniques and Applications, Kluwer Academic Publisher/Springer: Dordrecht, 2001. 
[66] P.J. Haines, Principles of Thermal Analysis and Calorimetry, Royal Society of Chemistry: Cambridge, 2002.

[67] H.P. Brack, D. Ruegg, H. Buhrer, M. Slaski, S. Alkan S, G.C. Schrer, J. Polym. Sci. Part B: Polym. Phys. 2004, 42, 2612.

[68] R. Gangopadhyay, P. Ghosh, Eur. Polym. J. 2000, 36, 1597.

[69] K. Zheng, L. Chen, Y. Li, P. Cui, Polym. Eng. Sci. 2004, 44, 1077.

[70] J.M. Gohil, A. Bhattacharya, P. Ray, J. Polym. Res. 2006, 13, 161.

[71] A. Ortega, E. Bucio, G. Burillo, Polym. Bull. 2007, 58, 565.

[72] M. Sen, M. Sari, Eur. Polym. J. 2005, 41, 1304.

[73] B. Wang , X. Xu, Z. Wang, S. Cheng, X. Zhang, R. Zhuo, Colloid Surface B 2008, 64, 34.

[74] L. Wang, W. Dong, Y. Xu. Carbohyd. Polym. 2007, 68, 626.

[75] Z. Dong, Z. Liu, B. Han, J. He, T. Jiang, G. Yang, J. Mater. Chem. 2002, 12, 3565.

[76] D.R. Bhumkar, V.B. Pokharkar, AAPS Pharmscitech. 2006, 7, E1.

[77] S. Phadnis, M. Patri, B.C. Chakraborty, P.K. Singh, P.C. Deb, J. Appl. Polym. Sci. 2005, 97, 1426.

[78] J. Chen, Y.C. Nho, O.H. Kwon, A.S. Hoffman, Radiat. Phys. Chem. 1999, $55,87$.

[79] J.Y. Park, M.H. Acar, A. Akthakul, W. Kuhlman, A.M. Mayes, Biomaterials 2006, $27,856$.

[80] N. Abidi, E. Hequet, S. Tarimala, Text. Res. J. 2006, 77, 668.

[81] Z.M. Liu, Z.K. Xu, J.Q. Wang, J. Wu, J.J. Fu, Eur. Polym. J. 2004, 40, 2077.

[82] V. Freger, J. Girlon, S. Belfer, J. Membr. Sci. 2002, 209, 283.

[83] E. Bucio, R. Aliev, G. Burillo, Polymer Bull. 2002, 47, 571.

[84] J.C. Ruiz, E. Bucio, R. Aliev, G. Burillo, Soc. Quím. Méx. 2004, 48, 208.

[85] A. Contreras-García, G. Burillo, R. Aliev, E. Bucio, Radiat. Phys. Chem. 2008, 77, 936.

[86] H.I. Meléndez-Ortiz, E. Bucio, Polymer Bull. 2008, 61, 619. 